\title{
A Loss of Parvalbumin-Containing Interneurons Is Associated with Diminished Oscillatory Activity in an Animal Model of Schizophrenia
}

\author{
Daniel J. Lodge, ${ }^{1,2,3}$ Margarita M. Behrens, ${ }^{4}$ and Anthony A. Grace ${ }^{1,2,3}$ \\ Departments of ${ }^{1}$ Neuroscience, ${ }^{2}$ Psychiatry, and ${ }^{3}$ Psychology, University of Pittsburgh, Pittsburgh, Pennsylvania 15260, and ${ }^{4}$ Department of Medicine, \\ Division of Geriatrics, University of California, San Diego, La Jolla, California 92093-0746
}

\begin{abstract}
Decreased GABAergic signaling is among the more robust pathologies observed postmortem in schizophrenia; however, the functional consequences of this deficit are still largely unknown. Here, we demonstrate, in a verified animal model of schizophrenia, that a reduced expression of parvalbumin (PV)-containing interneurons is correlated with a reduction in coordinated neuronal activity during task performance in freely moving rats. More specifically, methylazoxymethanol acetate (MAM)-treated rats display a decreased density of parvalbumin-positive interneurons throughout the medial prefrontal cortex (mPFC) and ventral (but not dorsal) subiculum of the hippocampus. Furthermore, the reduction in interneuron functionality is correlated with a significantly reduced gamma-band response to a conditioned tone during a latent inhibition paradigm. Finally, deficits in mPFC and ventral hippocampal oscillatory activity are associated with an impaired behavioral expression of latent inhibition in MAM-treated rats. Thus, we propose that a decrease in intrinsic GABAergic signaling may be responsible, at least in part, for the prefrontal and hippocampal hypofunctionality observed during task performance, which is consistently observed in animal models as well as in schizophrenia in humans. In addition, a deficit in intrinsic GABAergic signaling may be the origin of the hippocampal hyperactivity purported to underlie the dopamine dysfunction in psychosis. Such information is central to gaining a better understanding of the disease pathophysiology and alternate pharmacotherapeutic approaches.
\end{abstract}

Key words: parvalbumin; GABA; hippocampus; prefrontal cortex; schizophrenia; MAM

\section{Introduction}

Among the most robust pathologies observed in schizophrenia is a decrease in GABAergic signaling (Perry et al., 1979; Benes and Berretta, 2001; Heckers et al., 2002; Reynolds et al., 2002; Lewis et al., 2005; Benes et al., 2007; Lisman et al., 2008). Specifically, a decrease in glutamic acid decarboxylase (GAD)-1 mRNA and GAD-67 protein is observed postmortem throughout the cortex of schizophrenia patients (Akbarian et al., 1995; Volk et al., 2000; Hashimoto et al., 2003). These GABA deficits are largely restricted to the class of GABAergic interneurons containing the calcium binding protein parvalbumin (Lewis et al., 2005). These neurons synapse on the cell body or axon initial segment of glutamatergic neurons and thus are positioned to potently regulate pyramidal cell output. Furthermore, it is likely that a reduction in parvalbumin (PV) interneuron functionality would result not

Received Nov. 10, 2008; revised Jan. 16, 2009; accepted Jan. 21, 2009.

This work was supported by United States Public Health Service Grant MH57440 (A.A.G.) and a Young Investigator Award from National Alliance for Research on Schizophrenia and Depression-The Mental Health Research Association (D.J.L.). We thank Niki MacMurdo and Emily Mahar for their valuable contributions, Witold Lipski for his assistance with MATLAB, and Brian Lowry for the production, development, and support of the custom-designed electrophysiology software (Neuroscope).

Correspondence should be addressed to Daniel J. Lodge, Department of Neuroscience, University of Pittsburgh, A210 Langley Hall, Pittsburgh, PA 15260. E-mail: Lodge@pitt.edu.

DOI:10.1523/JNEUROSCI.5419-08.2009

Copyright $\odot 2009$ Society for Neuroscience $\quad$ 0270-6474/09/292344-11\$15.00/0 only in a decreased inhibitory control over pyramidal cell activity, but also a reduction in the coordinated activity of large brain networks. Indeed, substantial data demonstrate the importance of fast-spiking interneurons in the generation of cortical and hippocampal oscillatory potentials (Buzsáki et al., 1983; Whittington et al., 1995; Tamás et al., 2000; Bartos et al., 2002, 2007; Freund and Katona, 2007).

A failure in coordinated information processing between brain regions has been suggested to account for a wide range of deficits in schizophrenia, including both positive and cognitive symptoms (Haig et al., 2000; Ford et al., 2002; GonzalezHernandez et al., 2003; Spencer et al., 2003; Schmiedt et al., 2005; Cho et al., 2006; Uhlhaas et al., 2006; Basar-Eroglu et al., 2007; Uhlhaas et al., 2008). Furthermore, theta and gamma oscillations, induced in the prefrontal cortex during cognitive tasks, are reduced in schizophrenia patients, who consequently perform poorly on these tasks (Gonzalez-Hernandez et al., 2003; Schmiedt et al., 2005; Cho et al., 2006; Basar-Eroglu et al., 2007). Given evidence of a GABA dysfunction in schizophrenia patients and an association between GABA interneurons and coordinated neuronal activity, we examined the role of PV-containing interneurons in the regulation of synchronized medial prefrontal cortex (mPFC) and ventral hippocampus (vHipp) activity during task performance in an animal model of schizophrenia, namely the methylazoxymethanol acetate (MAM) G17 model. 


\section{Non-salient tone}

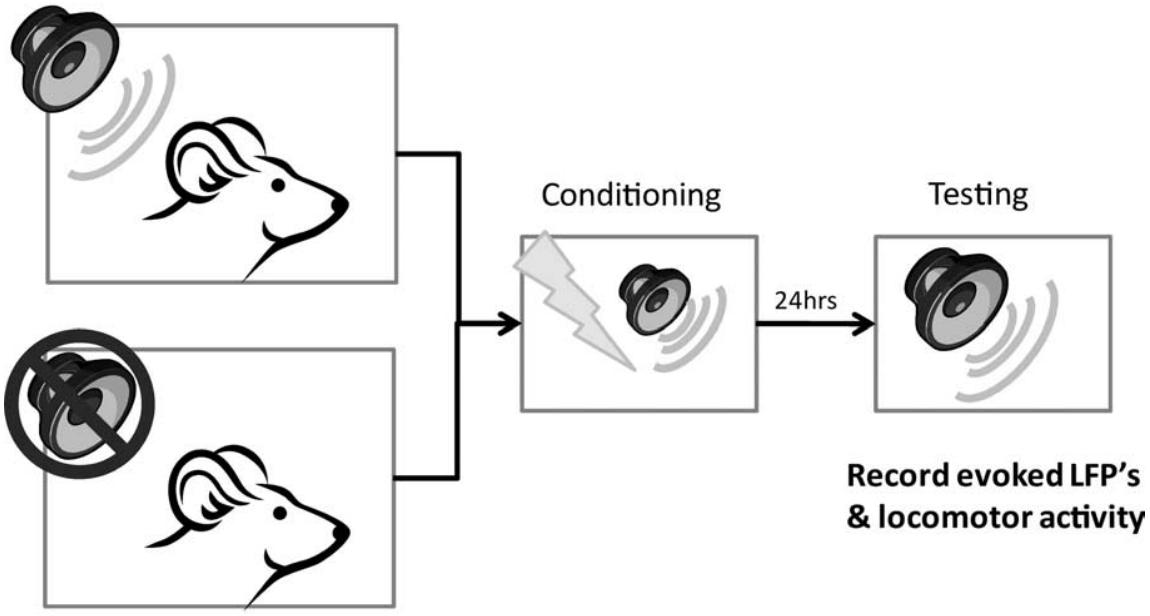

Record evoked LFP's

Figure 1. Summary of combined behavioral testing and stimulus-evoked LFP recordings in a latent inhibition paradigm. Rats were divided into two groups, with one group pre-exposed to a nonsalient tone and the other group having no tone presentation. All rats were subsequently exposed to a standard auditory fear conditioning procedure that involved a tone presentation that coterminated for the final second with a mild footshock. Twenty-four hours after conditioning, rats were placed in the conditioning chamber where the locomotor and tone-evoked responses to the conditioned tone were recorded.

\section{Prefrontal Cortex}

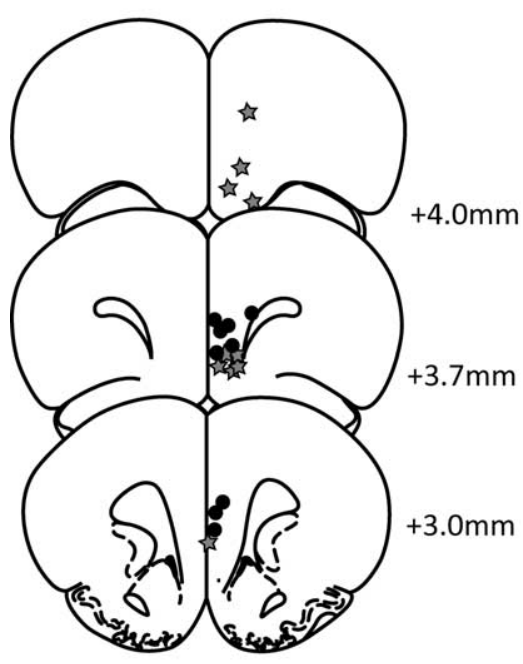

\section{Ventral Hippocampus}

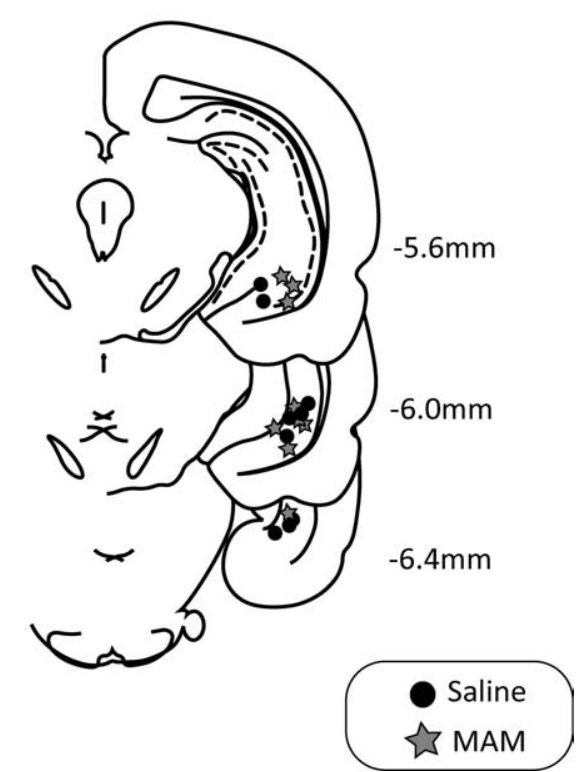

Figure 2. Histological localization of electrode sites within the prefrontal cortex and hippocampus. Numbers beside each plate represent approximate $A / P$ distance from bregma.

The MAM G17 model that we first developed (Grace and Moore, 1998) employs the administration of a mitotoxin, MAM, on gestational day (GD) 17 to pregnant rats to induce a developmental disruption (Grace and Moore, 1998; Talamini et al., 2000; Gourevitch et al., 2004; Le Pen et al., 2006; Moore et al., 2006). This model recapitulates a pathodevelopmental process leading to schizophrenia-like phenotypes in rodent offspring, which include anatomical changes (Moore et al., 2006; Penschuck et al., 2006), behavioral deficits (Grace and Moore, 1998; Talamini et al., 2000; Flagstad et al., 2004; Gourevitch et al., 2004; Moore et al., 2006) and altered neuronal information processing (Lavin et al., 2005; Goto and Grace, 2006; Lodge and Grace, 2007). Using this model, we now demonstrate that a decrease in intrinsic GABAergic functionality is associated with a diminished gamma band response during task performance in freely moving rats.

\section{Materials and Methods}

All experiments were performed in accordance with the guidelines outlined in the United States Public Health Service Guide for the Care and Use of Laboratory Animals and were approved by the Institutional Animal Care and Use Committee of the University of Pittsburgh.

Animals. MAM-treated rats were prepared as described previously (Moore et al., 2006; Lodge and Grace, 2007). In brief, timed pregnant female Sprague Dawley rats were obtained at GD15 and housed individually in plastic breeding tubs. MAM (diluted in saline, $20 \mathrm{mg} / \mathrm{kg}$, i.p.) was administered on GD17. Control dams received injections of saline $(1 \mathrm{ml} / \mathrm{kg}$, i.p.). Male pups were weaned on day 21 and housed in groups of two or three with littermates until $\sim 12$ weeks of age, at which time they were used for physiological or anatomical studies. All experiments were performed on multiple litters of MAM- and saline-treated rats.

Immunohistochemistry. Immunohistochemical studies were performed as described previously using commercially available antibodies (Behrens et al., 2007). Adult male rats were anesthetized with sodium pentobarbital (60 $\mathrm{mg} / \mathrm{kg}$, i.p.) and perfused transcardially with saline $(50 \mathrm{ml})$ followed by paraformaldehyde ( $100 \mathrm{ml}: 4 \% \mathrm{w} / \mathrm{v}$ in $0.1 \mathrm{~m}$ PBS). Rats were decapitated and their brains removed, postfixed for $24 \mathrm{~h}(4 \% \mathrm{w} / \mathrm{v}$ PFA in $0.1 \mathrm{M} \mathrm{PBS})$, and stored in $0.1 \mathrm{M}$ PBS until sectioning. Coronal sections (50 $\mu \mathrm{m})$ were obtained using a Vibratome. Sequential sections comprising the prefrontal region (bregma +5.0 to +2.0 ) and dorsal (bregma -1.5 to -4.5 ) and ventral (bregma -4.5 to -7.5 ) hippocampus (six slices per region) were used in fluorescence double immunohistochemistry to detect expression of GAD67 and parvalbumin as described previously (Behrens et al., 2007). Briefly, slices were treated with $1 \%$ sodium borohydride for 15 min before blocking in $10 \%$ normal goat serum (NGS) for $16 \mathrm{~h}$ at $4^{\circ} \mathrm{C}$. Primary antibodies [anti-GAD67 monoclonal 1:2000 (Millipore; MAB5406) and antiparvalbumin 1:3000 (Swant; PV28)] were applied in $2 \%$ NGS for $24 \mathrm{~h}$ followed by AlexaFluor-conjugated goat anti-rabbit (568) or goat anti-mouse (488) antibodies for $1 \mathrm{~h}$ at room temperature and mounted sequentially on glass slides using Vectashield.

Confocal microscopy and image analysis. Mounted slices were evaluated for fluorescence under settings for the AlexaFluor dyes 488 (Ex 488, Em $500-530$ band path) and 568 (Ex 543, Em 560 long path) on an LSM510 Meta multiphoton laser confocal microscope equipped with Argon and HeNe lasers and a 545 beam splitter, and using a $10 \times$ and $40 \times$ waterimmersion objectives. The settings of the confocal microscope were kept constant throughout the imaging such that fluorescence intensities could be compared between animals and imaging days (laser powers: $488=$ $21 \% ; 543=18 \%$ PMT $_{(488)}$ : gain $855 \mathrm{~V}$; offset: $0 ; \mathrm{PMT}_{(543)}$ : gain $775 \mathrm{~V}$; offset: 0 ). For slice imaging, each slice was imaged across the prefrontal and anterior and ventral hippocampal regions (two images per slice). Six 

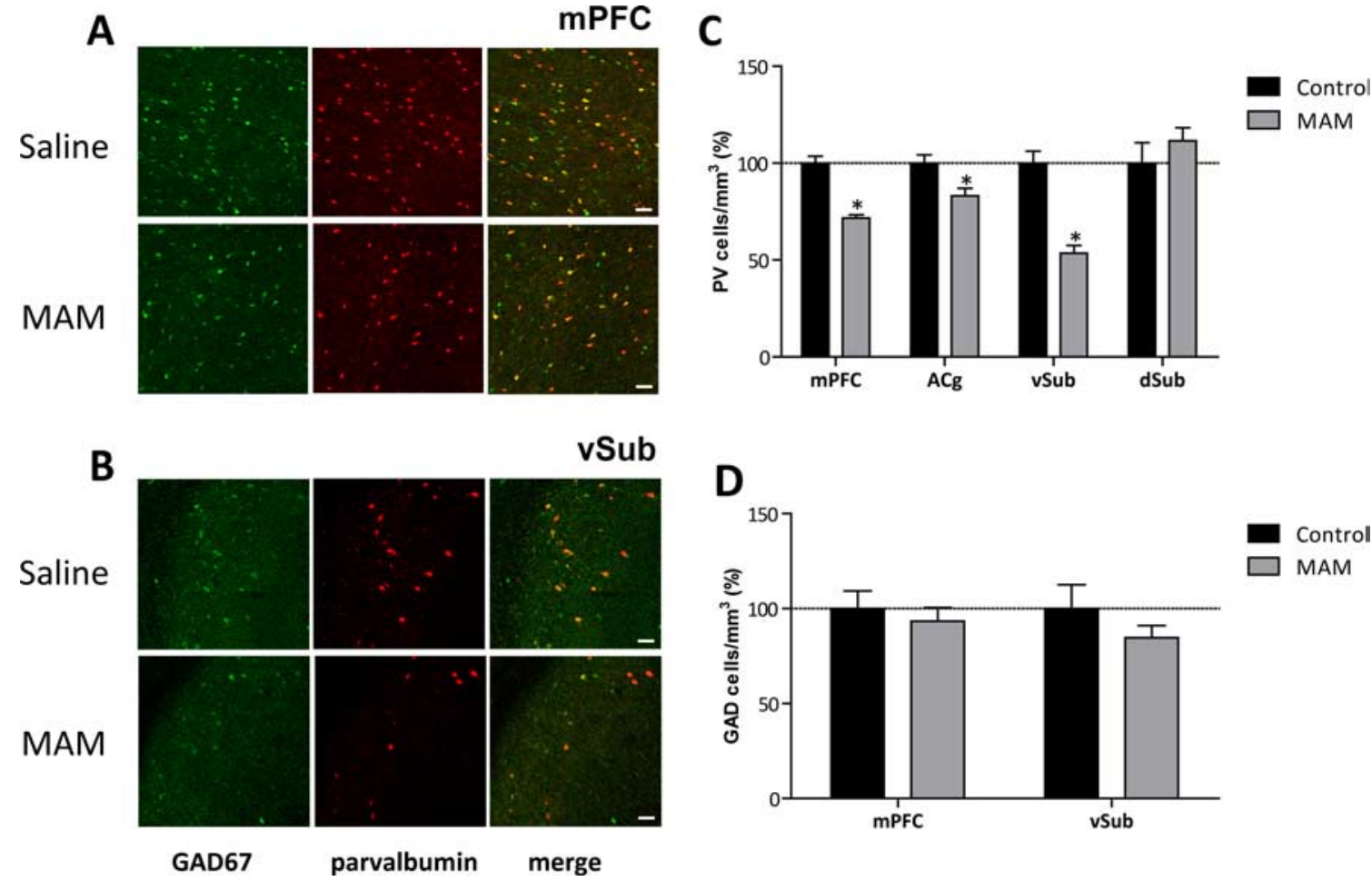

Figure 3. MAM-treated rats display a regionally specific reduction in the density of parvalbumin-positive neurons throughout the mPFC and the ventral vSub. Confocal $z$-stackimages of parvalbumin-(red) and GAD67(green) stained sections throughouttheprelimbicsubregion ofmPFC $(A)$ and vSub $(B)$ demonstratea decreasein PVinterneurons. The density of parvalbumin and GAD-67-positive/parvalbumin-negativeneurons aredepicted in Cand D respectively. * Statistically significant difference from control (prenatal saline administration) $(p<0.05$, one-way ANOVA, Tukey posthoc, $n=4-5$ rats/group). Scale bars, $50 \mu \mathrm{m}$.

A

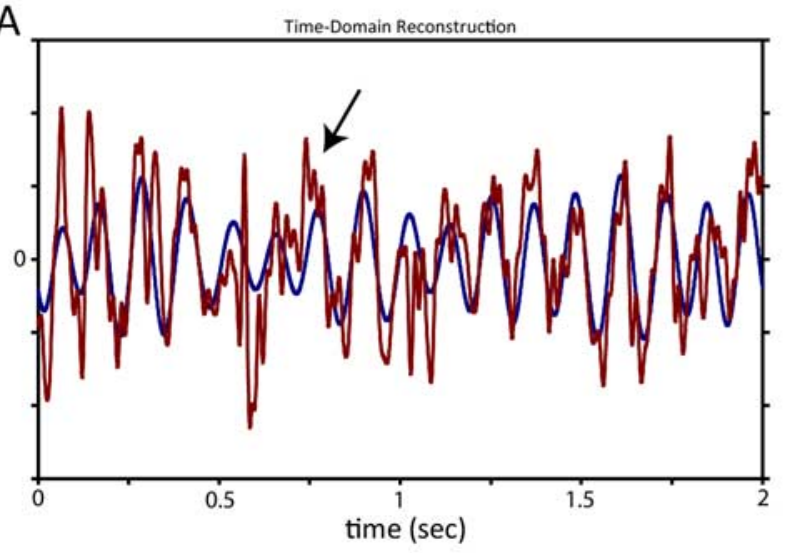

C

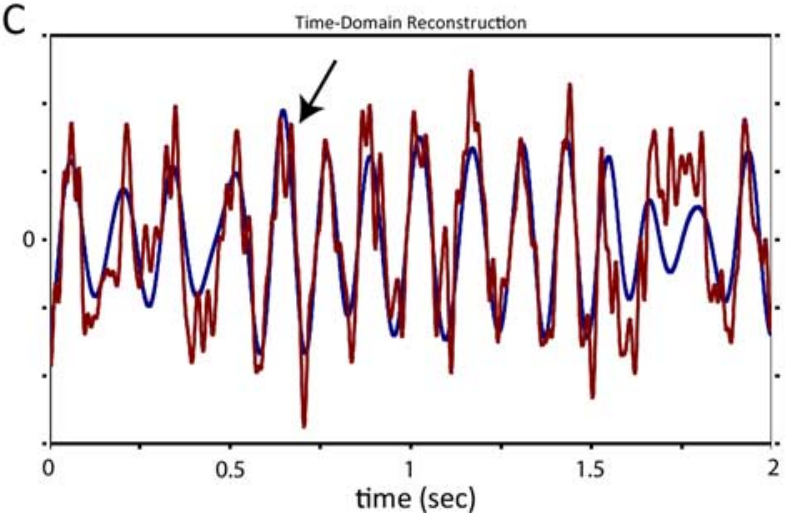

B

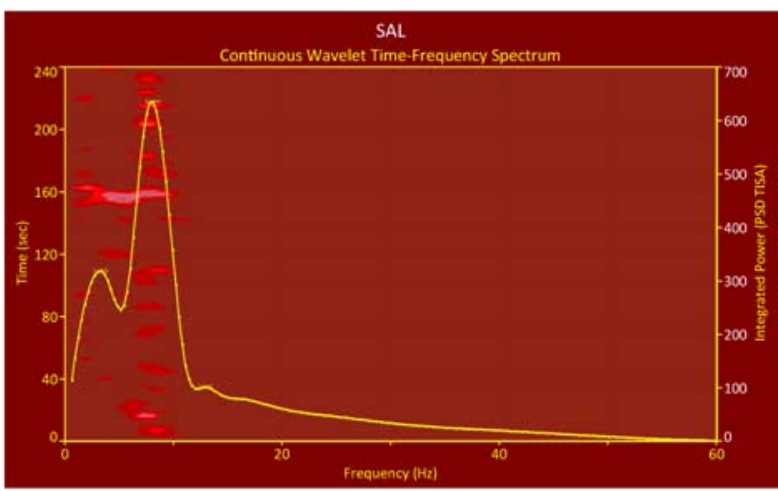

D

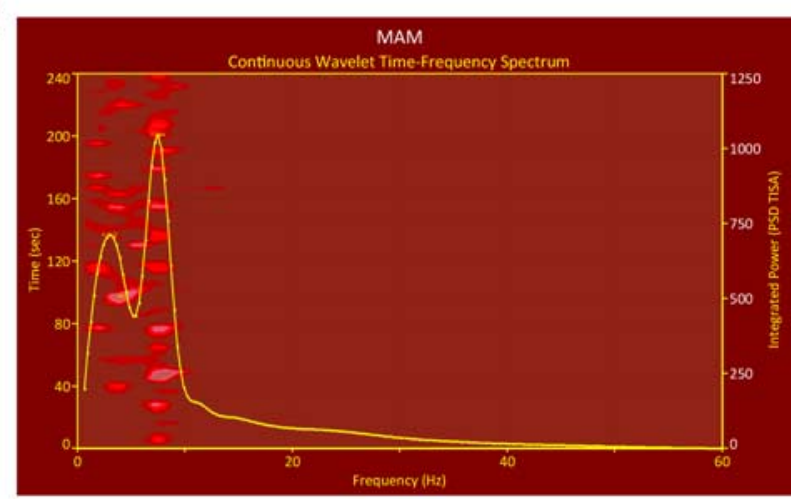

Figure 4. Spontaneous local field potential oscillations throughout the ventral hippocampus of saline- $(\boldsymbol{A}, \boldsymbol{B})$ and MAM- $(\boldsymbol{C}, \boldsymbol{D})$ treated rats. A time-domain reconstruction $(\boldsymbol{A}, \boldsymbol{C})$ demonstrates the predominant theta rhythm (blue line), a hallmark of oscillatory hippocampal activity, overlaid on a $2 \mathrm{~s}$ epoch of spontaneous activity (red line). The arrows indicate periods of high-frequency oscillations "riding" the theta wave. A continuous wavelet time-frequency spectrum $(\boldsymbol{B}, \mathbf{D})$ demonstrates the power (TISA), indicated by the red gradient contour, across frequency for a 4 min period ( $y 1$ axis). The global wavelet spectrum for the entire time range is depicted by the yellow line, plotted against the $y 2$ axis. 
A

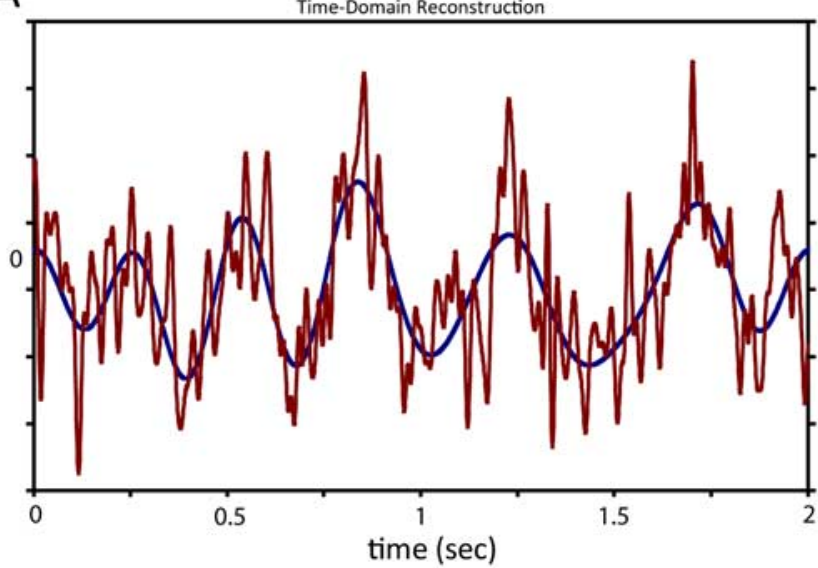

C

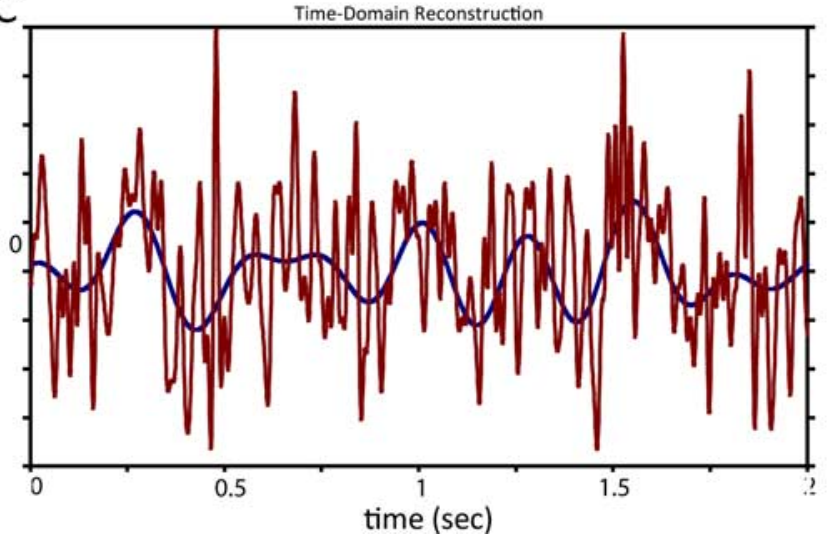

B

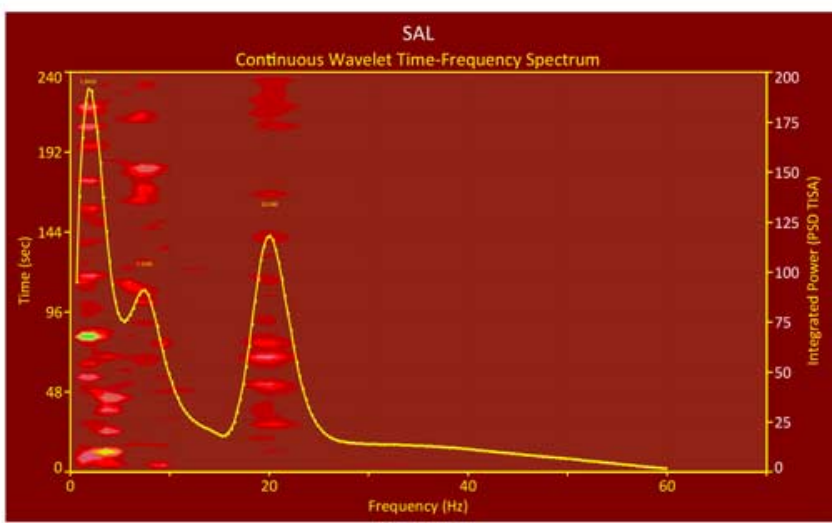

D

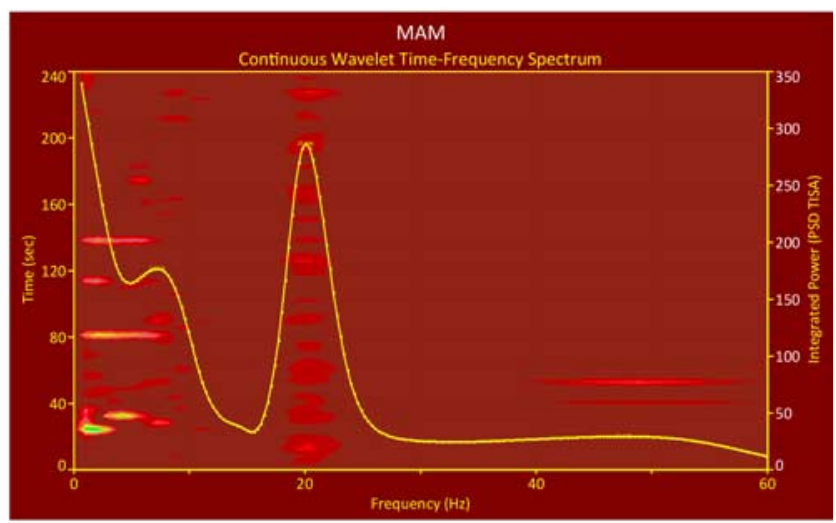

Figure 5. Spontaneous local field potential oscillations throughout the medial prefrontal cortex of saline- $(\boldsymbol{A}, \boldsymbol{B})$ and $M A M-(C, D)$ treated rats. A time-domain reconstruction $(A, C)$ demonstrates a low-frequency (delta) rhythm (blue line) overlaid on a 2 s epoch of spontaneous activity (red line). Strong periodic high-frequency oscillations $(\sim 20 \mathrm{~Hz})$ are present in the raw trace. $A$ continuous wavelet time-frequency spectrum $(\boldsymbol{B}, \boldsymbol{D})$ demonstrates the power (TISA), indicated by the red gradient contour, across frequency for a 4 min period ( $y 1$ axis). The global wavelet spectrum for the entire time range is depicted by the yellow line, plotted against the $y 2$ axis.

slices were imaged per animal. For fluorescence intensity quantification, a $z$-stack of eight images was obtained (corresponding to $1.4 \mu \mathrm{m}$ on the $z$-axis) using MetaMorph analysis software. All PV neurons in the images were analyzed for their parvalbumin and GAD67 content. For counting $\mathrm{PV}$ and non-PV interneurons, slices were imaged every $5 \mu \mathrm{m}$ across the entire slice and then collapsed to obtain one image of the resulting $z$-stack (area: $921.5 \mu \mathrm{m}^{2}$ ). All PV-positive cells as well as all GAD67-positive cells in the images were counted. Since all PV-positive also contain GAD, the cell numbers reported for GAD67 are only those GAD67-positive cells that did not express detectable levels of PV. The density of neurons $/ \mathrm{mm}^{3}$ for each animal region was obtained by calculating the number of neurons/slice using Abercrombie's correction (Abercrombie, 1946) and a neuron diameter of $12 \mu \mathrm{m}$. Slice preparation and imaging was performed by a researcher unaware of treatment groups.

Survival surgery. All survival surgical procedures were performed under general anesthesia in a semisterile environment. Briefly, male rats $(275-350 \mathrm{~g})$ were anesthetized with sodium pentobarbital $(60 \mathrm{mg} / \mathrm{kg}$, i.p.) and placed in a stereotaxic apparatus using blunt atraumatic ear bars. Custom length polyimide-insulated stainless steel wire electrodes (Plastics One: MS303/2-AIU) were lowered into the right medial prefrontal cortex [anterior/posterior (A/P) $+3.2 \mathrm{~mm}$ from bregma, medial/ lateral $(\mathrm{M} / \mathrm{L})-0.5 \mathrm{~mm}$ from midline, dorsal/ventral $(\mathrm{D} / \mathrm{V})-4.0 \mathrm{~mm}$ ventral of skull surface) and right ventral hippocampus (A/P $-6.0 \mathrm{~mm}$ from bregma, $\mathrm{M} / \mathrm{L}-5.3 \mathrm{~mm}$ from midline, $\mathrm{D} / \mathrm{V}-7.0 \mathrm{~mm}$ ventral of skull surface]. Noninsulated stainless steel ground wires were wrapped around a skull screw. The electrodes were fixed in place with dental cement and a total of five anchor screws. Once the cement was completely solid, the wound was sutured and the rat removed from the ste- reotaxic frame and monitored closely until conscious. Rats received antibiotic treatment (gentamicin $3 \mathrm{mg} / \mathrm{kg}$, s.c.) and postoperative analgesia (Children's Tylenol syrup in softened rat chow; $5 \% \mathrm{v} / \mathrm{w}$ ) ad libitum for $48 \mathrm{~h}$. Rats were housed with a reverse light/dark cycle (lights on 7:00 P.M. to 7:00 A.M.) for at least 2 weeks before conducting behavioral experiments.

Latent inhibition. All behavioral testing was performed during the active period of the diurnal cycle (between 7:00 A.M. and 7:00 P.M.). A representation of the behavioral paradigm is presented in Figure 1. Rats were brought to the testing area and the chronically implanted intracranial electrodes were connected to a CyberAmp 320 preamplifier (Axon Instruments) via a four channel commutator (Plastics One) and the rats placed in an open field arena (Coulbourn Instruments) where baseline oscillatory activity was recorded for a period of $5 \mathrm{~min}$. The output of the preamplifier was amplified $(\times 1000)$, filtered (low pass: $1 \mathrm{kHz})$ and digitized $(1 \mathrm{kHz})$ for off-line analysis. Rats were divided into two groups, with one group pre-exposed to a $2 \mathrm{~s}$ tone for 50 trials with a pseudorandom intertrial interval (range 15-45 s) and tone-evoked changes in local field potentials were recorded. The other group had no tone presentation but local field potentials (LFPs) were recorded for 50 trials. All rats were subsequently exposed to a standard auditory fear conditioning procedure that involved a $2 \mathrm{~s}$ tone presentation that coterminated for the final second with a mild $(0.6 \mathrm{~mA})$ footshock, administered through stainless steel bars in the floor of the chamber (Coulbourn Instruments). Conditioned pairings were performed 10 times with a pseudorandom intertrial interval (45-100 s). Rats were then returned to the animal colony room. $24 \mathrm{~h}$ after conditioning, rats were placed in the conditioning chamber where LFP responses and spontaneous locomotor activity in the $x, y$ plane 

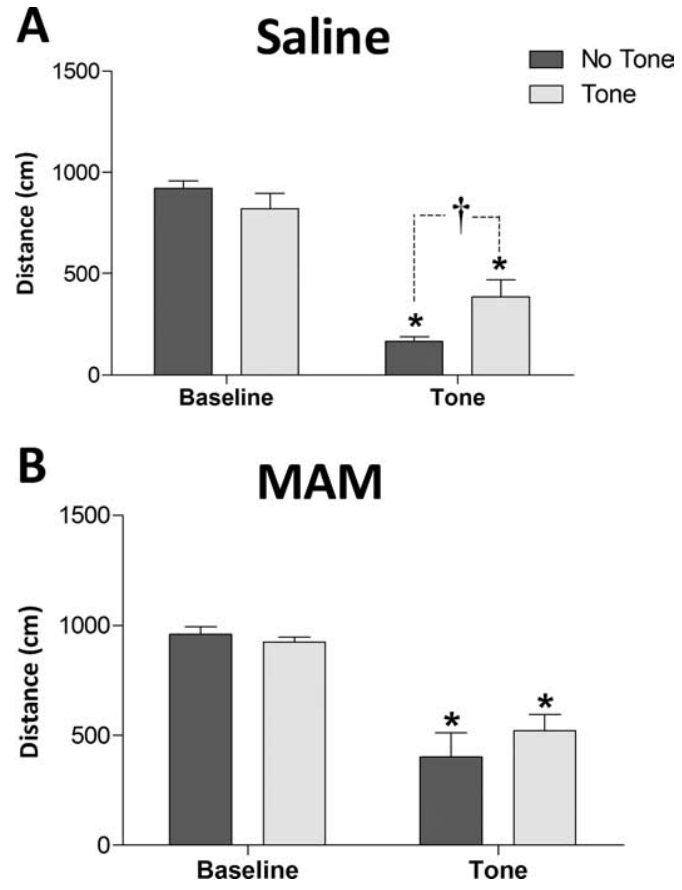

Figure 6. MAM-treated rats display deficits in latent inhibition. Rats that had received a mild footshock paired with a tone display a robust decrease in locomotor activity in response to the conditioned tone (dark bars). Control rats $(\boldsymbol{A})$ that had been exposed to the tone before the conditioning period displayed robust latent inhibition, i.e., an attenuated locomotor response to the tone $(\boldsymbol{A}$, light bars). In contrast, MAM-treated rats $(\boldsymbol{B})$ display a deficit in latent inhibition with the previous exposure to the tone having no significant effect on the locomotor response to the conditioned stimulus ( $\boldsymbol{B}$, light bars). ${ }^{*}$ Significant difference compared with baseline; ${ }^{\dagger}$ significant difference between tone and no tone pre-exposure ( $p<0.05$, two-way RM ANOVA with Holm-Sidak post hoc: $n=5$ rats/group).

was monitored for 5 min by beam breaks and recorded with TruScan software (Coulbourn Instruments). All rats were then exposed to 10 presentations of a $2 \mathrm{~s}$ tone with a pseudorandom intertrial interval (45$100 \mathrm{~s}$ ) where locomotion (for a period of $5 \mathrm{~min}$ ) and tone-evoked LFP responses were recorded.

Histology. At the cessation of the experiments all rats were killed by a lethal dose of anesthetic (sodium pentobarbital, $120 \mathrm{mg} / \mathrm{kg}$, i.p.). Rats were decapitated and their brains removed, fixed for at least $48 \mathrm{~h}(8 \% \mathrm{w} / \mathrm{v}$ paraformaldehyde in PBS), and cryoprotected (25\% w/v sucrose in PBS) until saturated. Brains were sectioned (60 $\mu \mathrm{m}$ coronal sections), mounted onto gelatin-chrome alum coated slides and stained with cresyl violet for histochemical verification of electrode sites. All histology was performed with reference to a stereotaxic atlas and electrode locations are presented in Figure 2 (Paxinos and Watson, 1986).

Electrophysiology analysis. Analysis of spontaneous local field potential activity was performed using AutoSignal software (Seasolve Software). In brief, LFP oscillatory activity was filtered $(0-58 \mathrm{~Hz})$, detrended (mean subtracted and linear trend removed) and analysis across time was performed on a 4 min period of spontaneous recording using a continuous wavelet (Morlet) time-frequency analysis that reported integrated power (time-integral-squared amplitude, TISA) across time. In addition, $2 \mathrm{~s}$ representative epochs were examined using a Fourier time-domain reconstruction to graphically demonstrate the principle frequency components of the LFP signal, whereas $10 \mathrm{~s}$ epochs were Fourier filtered to quantify cortical oscillations in the theta $(4-12 \mathrm{~Hz})$ and gamma $(30-55$ $\mathrm{Hz}$ ) bands. Evoked changes in LFP responses were examined using chronux analysis routines (www.chronux.org) in MATLAB (The MathWorks). In brief, continuous process multitaper time-frequency spectral analyses were performed on auditory-evoked LFP signals. Tone-evoked changes in power were compared using peak analyses (GraphPad Prism) and the maximum increases in power after the tone were determined for each rat and are expressed as a percentage of baseline. All data are represented as the mean \pm SEM unless otherwise stated.
Materials. MAM was purchased from Midwest Research Institute and Pentobarbital sodium was obtained from Ovation Pharmaceuticals. Mouse monoclonal anti-GAD67 was obtained from Millipore, and rabbit anti-parvalbumin was obtained from Swant. All other chemicals and reagents were of either analytical or laboratory grade and purchased from various suppliers.

\section{Results \\ Immunohistochemistry}

Rats that received GD17 MAM injections exhibited a regionally selective reduction in the density of $\mathrm{PV}$-positive neurons throughout the mPFC (Fig. 3a,c) [saline (Sal): $1712 \pm 62 \mathrm{PV}$ cells/mm ${ }^{3}$, MAM: $1226.9 \pm 29$ PV cells $/ \mathrm{mm}^{3}, n=5, p<0.001$, one-way ANOVA, Tukey post hoc) and ventral subiculum of hippocampus (Fig. $3 b, c)$ (Sal: $2346.3 \pm 146$ cells $/ \mathrm{mm}^{3}$, MAM: $1255.4 \pm 96$ cells $/ \mathrm{mm}^{3}, n=4, p<0.001$, one-way ANOVA, Tukey post hoc) with no significant differences in the number of GAD-67-positive/parvalbumin-negative neurons in either the mPFC (Fig. 3a,d) (Sal: $2267+/-211$ cells $/ \mathrm{mm}^{3}$, MAM: 2117 $+/-152$ cells $\left./ \mathrm{mm}^{3}, n=5\right)$ or ventral subiculum of hippocampus (Fig. 3b,d) (Sal: $5019+/-633$ cells/ $\mathrm{mm}^{3}$, MAM: $4249+/-$ 273 cells $\left./ \mathrm{mm}^{3}, n=4\right)$. These differences in PV cell density were not observed in the dorsal subiculum of the hippocampus (Fig. 3c) (Sal: 1870.4 \pm 198.6 PV cells/mm ${ }^{3}$, MAM: 2087.6 $\pm 124.7 \mathrm{PV}$ cells $\left./ \mathrm{mm}^{3}, n=5, p=0.381\right)$ while a small decrease in PV cell density was observed throughout the anterior cingulate cortex (Fig. 3c) (Sal: $2133.4 \pm 91 \mathrm{PV}$ cells $/ \mathrm{mm}^{3}$, MAM: $1775.7 \pm 83 \mathrm{PV}$ cells $/ \mathrm{mm}^{3} n=5, p=0.02$, one-way ANOVA, Tukey post hoc). In addition, there were no significant differences in fluorescence intensity for either GAD-67 [mPFC (arbitrary units)-Sal: $44.0 \pm$ 2.3, MAM: $52.3 \pm 3.6$; ventral subiculum of the hippocampus (vSub)-Sal: $43.5 \pm 2.5$, MAM: $42.1 \pm 3.0, n=4-5$ ) or PV (mPFC-Sal: $190.2 \pm$ 8.6, MAM: $185.7 \pm 5.2$; vSub-Sal: $88.0 \pm 9.1$, MAM: $72.7 \pm 2.0, n=4-5)$.

\section{Spontaneous LFP activity}

Spontaneous oscillatory activity was observed in baseline recordings from the mPFC and vHipp of both MAM- and saline-treated rats (Figs. 4, 5). Continuous wavelet time-frequency spectral analysis demonstrated a predominant peak in the theta range (peak $\sim 7.5 \mathrm{~Hz}$ ) and a secondary peak in the delta range (peak $\sim 2$ $\mathrm{Hz}$ ) throughout the vHipp of both saline- (Fig. $4 a, b$ ) and MAM(Fig. $4 c, d$ ) treated rats. In contrast, mPFC local field potentials displayed significantly less oscillatory activity (Fig. 5) with a prominent low-frequency peak in the delta range $(<3 \mathrm{~Hz})$, a secondary peak in the theta range (peak $\sim 7.5 \mathrm{~Hz}$ ) and a tertiary high-frequency peak in the beta/gamma range (peak $\sim 20 \mathrm{~Hz}$ ) in both saline- (Fig. 5a,b) and MAM- (Fig. $5 c, d$ ) treated rats. Interestingly, there were no significant differences between MAM and saline rats in spontaneous activity throughout the mPFC [theta [power spectral density (PSD)-TISA: $\mu \mathrm{V}^{2} \cdot \mathrm{Hz}^{-1}$ ] Sal: $92.7 \pm$ 11.7, MAM: $102.9 \pm 13.5$; gamma (PSD-TISA: $\mu \mathrm{V}^{2} \cdot \mathrm{Hz}^{-1}$ ) Sal: $27.5 \pm 3.9$, MAM: $34.9 \pm 7.6, n=9-10$ ] or vHipp [theta (PSDTISA: $\left.\mu \mathrm{V}^{2} \cdot \mathrm{Hz}^{-1}\right)$ Sal: $649.5 \pm 154.0$, MAM: $433.4 \pm 156.9$; gamma (PSD-TISA: $\mu \mathrm{V}^{2} \cdot \mathrm{Hz}^{-1}$ ) Sal: $186.4 \pm 79.8$, MAM: $77.92 \pm 18.7, n=9-10$ ]; however, high between subject variability, due to electrode location, may mask any differences between saline and MAM-treated rats.

\section{Latent inhibition}

Rats were prepared for behavioral testing and simultaneous evoked field recordings (Fig. 1). Consistent with previous literature in untreated rats, the presentation of a conditioned tone 
mPFC theta
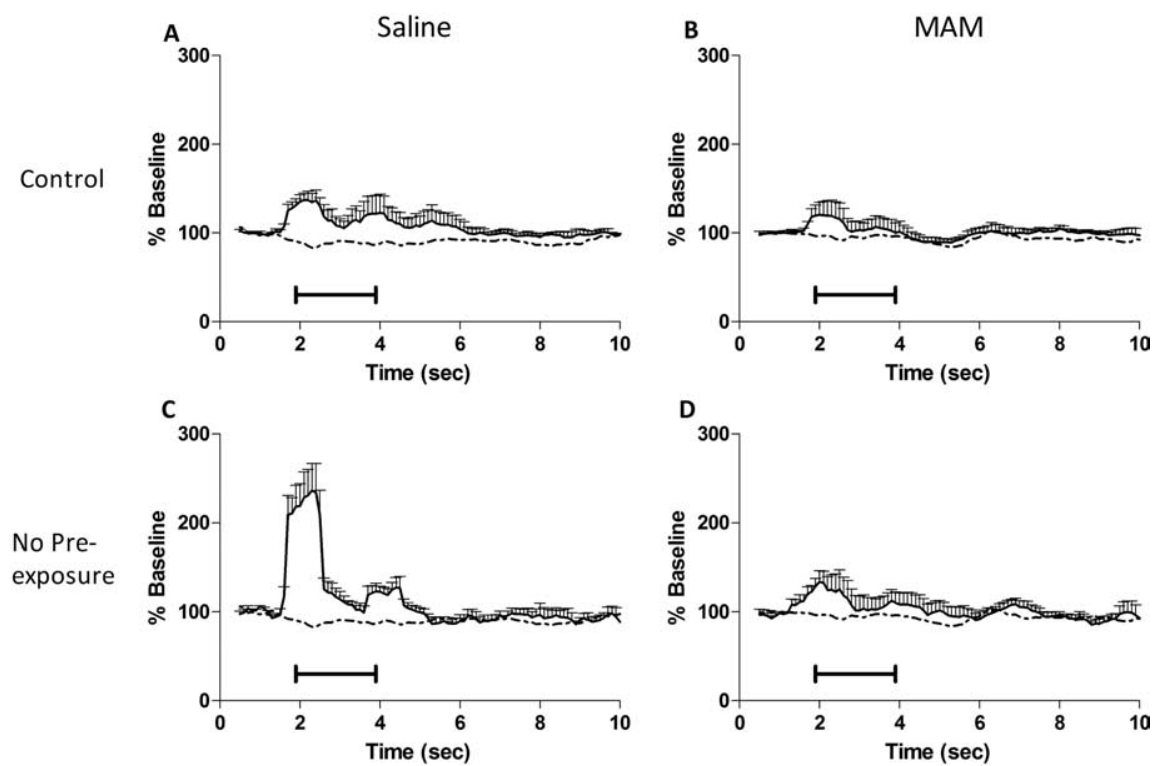

D
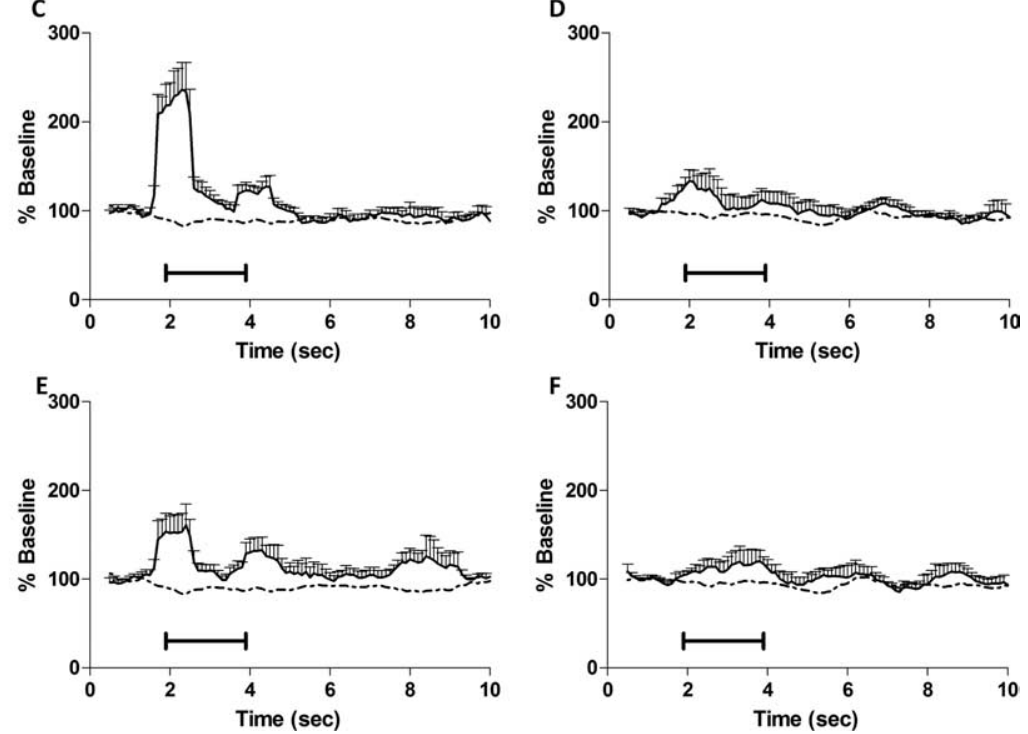

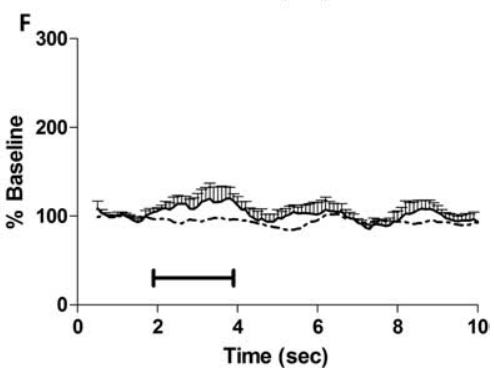

Figure 7. $\mathrm{mPFC}$ theta response to the conditioned tone is reduced in MAM-treated rats. Multitaper spectral analyses of tone-evoked local field potential responses demonstrate that tone presentation alone induces a mild increase in prefrontal theta $(4-12 \mathrm{~Hz})$ oscillations in both saline $(\boldsymbol{A})$ and MAM $(\boldsymbol{B})$ rats. Control rats that had received a mild footshock paired with a tone display a massive and sustained increase in theta activity evoked by the conditioned tone $(\boldsymbol{C})$, that is attenuated in rats that had been exposed to the tone before the conditioning period $(\boldsymbol{E})$. In contrast, MAM-treated rats did not display a robust response to the conditioned tone either in rats with $(\boldsymbol{F})$ or without $(\boldsymbol{D})$ previous tone exposure. The horizontal line depicts the 2 s tone presentation whereas the dashed line represents the control response (i.e., no tone presentation); $n=3-5$ rats/group.

evoked a significant decrease in locomotor activity in both saline(Fig. 6a) [baseline: $920.3 \pm 37.0 \mathrm{~cm}$, tone: $164.3 \pm 23.0 \mathrm{~cm}, n=$ $4, p<0.05$, two-way repeated-measures (RM) ANOVA with Holm-Sidak post hoc] and MAM- (Fig. 6b) (baseline: $960.2 \pm$ $33.8 \mathrm{~cm}$, tone: $403.2 \pm 108.7 \mathrm{~cm}, n=5, p<0.05$, two-way RM ANOVA with Holm-Sidak post hoc) treated rats. Control rats that had been exposed to the tone before the conditioning period displayed robust latent inhibition; i.e., an attenuated conditioned response to the tone (Fig. 6a) (no tone pre-exposure: $164.3 \pm$ $23.0 \mathrm{~cm}$, tone pre-exposure: $385.6 \pm 81.9 \mathrm{~cm}, n=4, p<0.05$, two-way RM ANOVA with Holm-Sidak post hoc). In contrast and consistent with previous data, MAM-treated rats displayed a deficit in latent inhibition, with previous exposure to the tone having no significant effect on the subsequent locomotor response to the conditioned stimulus (Fig. 6b) [no tone pre-exposure: $403.2 \pm 108.7 \mathrm{~cm}$, tone pre-exposure: $520.8 \pm 74.6 \mathrm{~cm}, n=$ 5 , not significant ( $\mathrm{ns}$ ), two-way RM ANOVA]. It should be noted that MAM-treated rats display a reduced response to the conditioned tone when compared with saline rats that is not associated with alterations in auditory processing since MAM treated rats display a lower threshold for responding to auditory stimuli (Moore et al., 2006).

\section{Evoked LFP activity}

Electrophysiological recordings from the $\mathrm{mPFC}$ and vHipp were performed in freely behaving rats during latent inhibition training and testing. A representative trace of tone-evoked activity is presented in supplemental Figure 1, available at www. jneurosci.org as supplemental material. Multitapered spectral analysis of toneevoked activity demonstrated a slight response to the tone presentation before conditioning. In both saline and MAMtreated rats, the unconditioned tone tended to increase mPFC theta (Fig. 7a,b) (Sal 148.6 \pm 16.8 , MAM $129.9 \pm 15.8 \%$ of baseline), mPFC gamma (see Fig. 9a,b) (Sal 124.6 \pm 14.3, MAM $115.5 \pm 5.5 \%$ of baseline) and vHipp gamma (see Fig. $10 a, b)$ (Sal $142.7 \pm 18.1$, MAM $123.2 \pm$ $11.3 \%$ of baseline) with no observable change in vHipp theta (Fig. 8a,b) (Sal $106.5 \pm 6.5$, MAM $110.4 \pm 9.7 \%$ of baseline). Interestingly, after fear conditioning in saline-treated rats, the conditioned tone induced a massive increase in $\mathrm{mPFC}$ theta (Fig. $7 c)(237.8 \pm 36.3 \%$ of baseline, $p<$ 0.05 from no tone, two-way ANOVA with Holm-Sidak post hoc) and mPFC gamma (Fig. 9c) (198.6 $\pm 48.7 \%$ of baseline, $p<$ 0.05 from no tone, two-way ANOVA with Holm-Sidak post hoc) without significantly altering evoked theta (Fig. 8c) $(132.0 \pm 16.1 \%$ of baseline, ns, two-way ANOVA) or gamma (Fig. 10c) (169.7 \pm $20.0 \%$ of baseline, ns, two-way ANOVA) in the vHipp. In contrast, the conditioned stimuli did not significantly alter oscillatory activity in MAM-treated rats and was thus significantly attenuated compared with saline-treated rats (Fig. $7 d$ ) (mPFC theta $146.1 \pm 18.2$ ) (Fig. $9 d$ ) (mPFC gamma $139.1 \pm 12.0$ ) (Fig. 10d) (vHipp gamma 116.4 \pm 6.0 , all $p<0.05$ from responses in control rats, two-way ANOVA with Holm-Sidak post hoc). There were no significant differences between saline and MAM rats in vHipp theta activity (Fig. $8 d$ ) $(124.4 \pm 7.2$; ns, two-way ANOVA). It is important to note that there were no statistically significant differences in gamma power preceding the onset of the tone in either the mPFC or vHipp of conditioned MAM or saline rats.

Control rats that had been exposed to the tone before the conditioning period demonstrated a significantly attenuated conditioned response to the tone (Fig. 11). Specifically, a reduced mPFC oscillatory response was observed in the theta band (Fig. $7 e)(147.2 \pm 19.8 \%$ of baseline, $p<0.05$ from nontone preexposed rats, two-way ANOVA with Holm-Sidak post hoc) whereas no significant effect was observed in mPFC gamma (Fig. $9 e)(146.5 \pm 16.0 \%$ of baseline, ns, two-way ANOVA $)$ or vHipp theta (Fig. $8 e)(111.8 \pm 8.8 \%$ of baseline, ns, two-way ANOVA) or gamma (Fig. 10e) $(147.6 \pm 21.3 \%$ of baseline, ns, two-way ANOVA). Pre-exposure to the conditioned stimulus did not significantly alter oscillatory activity in MAM-treated rats (Fig. $7 f$ ) (mPFC theta $130.9 \pm 16.6$ ) (Fig. $8 f$ ) (vHipp theta $116.8 \pm 12.3$ ) 
(Fig. 9f) (mPFC gamma 126.1 \pm 9.8) (Fig. 10f) (vHipp gamma $127.2 \pm 5.9$, all ns, two-way ANOVA).

\section{Discussion}

These data demonstrate that MAMtreated rats display a pathological reduction in PV interneuron density throughout the mPFC and vHipp that is associated with altered prefrontal and hippocampal activation during task performance. Furthermore, the degree of activation of cortical assemblies was correlated with the behavioral response to a conditioned tone in a latent inhibition paradigm. Together, we propose that a decrease in intrinsic GABAergic signaling may be responsible, at least in part, for the prefrontal and hippocampal hypofunctionality consistently disrupted in animal models as well as in schizophrenia patients.

A regionally selective decrease in PV interneuron density was observed throughout the medial prefrontal cortex and ventral (but not dorsal) subiculum of the hippocampus in MAM-treated rats. This decrease appears specific for PV containing interneurons and is not associated with changes in GAD-67 interneurons or total neuronal number (Moore et al., 2006). This is consistent with previous reports demonstrating reduced PV interneuron functionality throughout the cortex, including dorsolateral prefrontal cortex, as well as throughout all of the subregions of the hippocampus in postmortem brains of schizophrenia patients (Woo et al., 1998; Zhang and Reynolds, 2002; Lewis et al., 2005). A decrease in parvalbumincontaining interneurons is also consistently observed across a variety of animal models of schizophrenia, including the MAM model (Penschuck et al., 2006), the chronic phencyclidine model (Abdul-Monim et al., 2007), in rats reared in isolation (Harte et al., 2007) and the amygdala activation model (Berretta et al., 2004). It is important to note that the decrease in PV immunoreactivity and mRNA expression noted in postmortem studies or animal models is believed to be associated with a reduced expression of PV rather than an actual decrease in the number of interneurons (Hashimoto et al., 2003).

Given their strong perisomatic innervation of glutamatergic neurons, parvalbumin interneurons are situated to potently regulate pyramidal cell output (Lewis and Lund, 1990). Thus it is likely that a reduction in PV interneuron functionality would interfere with the coordinated activity of cortical assemblies that drive synchronous activity in the gamma band across brain networks. Indeed, the present study demonstrates that MAMtreated rats display a deficit in the ability to activate cortical and hippocampal assemblies during a latent inhibition paradigm. Thus, after tone-shock pairing in saline-treated rats, the conditioned tone induced a sustained increase in low-frequency (theta) activity and a transient increase in high-frequency (gamma) power in the $\mathrm{MPFC}$, without significantly altering coordinated activity in the vHipp. This is consistent with previous work showing that fast-spiking interneurons display a robust, transient increase in firing rate in response to a conditioned tone, whereas the response observed in pyramidal neurons is more sustained, being time locked to the duration of conditioned stimulus (CS) presentation (Baeg et al., 2001). Furthermore, it has been suggested that the transient, high-frequency activation of interneurons may direct attention toward the CS while the more sustained, pyramidal cell activity may play a role in the anticipation of the unconditioned aversive stimuli (Baeg et al., 2001).

Interestingly, the present study demonstrated that the fearconditioned tone did not significantly alter oscillatory activity in MAM-treated rats compared with the robust responses observed in saline-treated rats. Given the role for fast-spiking interneurons in the generation of gamma oscillatory potentials (Buzsáki et al., 1983; Whittington et al., 1995; Tamás et al., 2000; Bartos et al., 2002, 2007; Freund and Katona, 2007), we suggest that this is likely associated with the observed decrease in PV interneuron density. Indeed, the deficit in gamma activity in the MAMtreated rats was task-independent and observed in both the $\mathrm{mPFC}$ and vHipp; regions that exhibited significant reductions in PV neuron density. These data are consistent with studies demonstrating a clear correlation between PV immunoreactivity and the generation of gamma rhythms in entorhinal cortical slices from lysophosphatidic acid 1 receptor knock-out mice as well as 
mPFC gamma
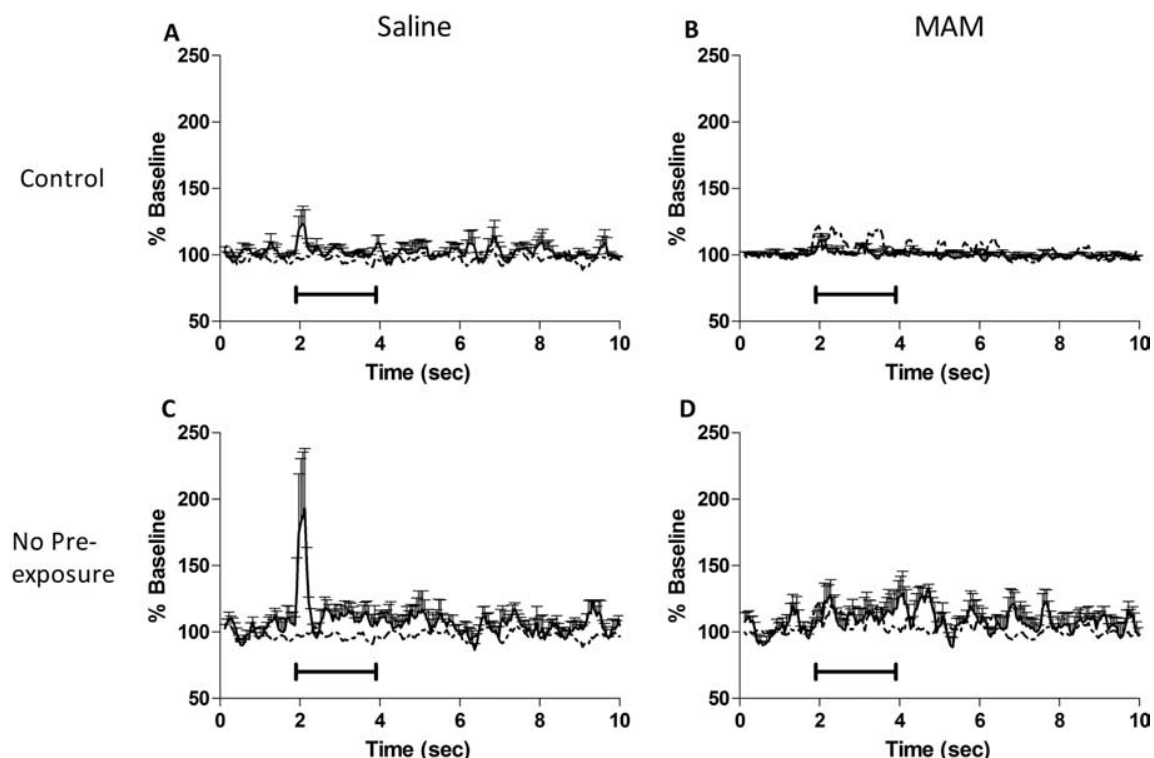

D
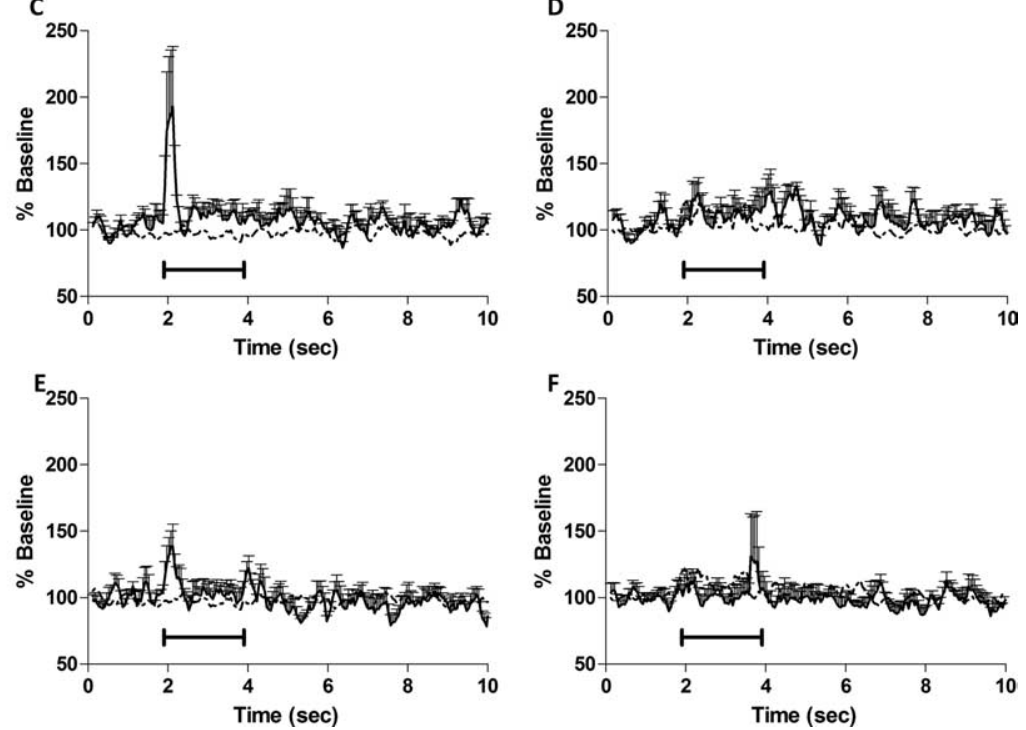

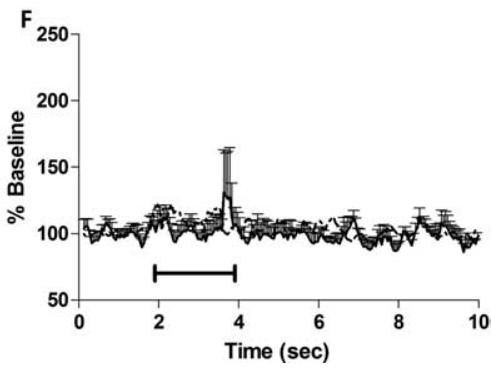

Figure 9. High-frequency $\mathrm{mPFC}$ responses to the conditioned tone are reduced in MAM-treated rats. Multitaper spectral analyses of tone-evoked local field potential responses demonstrate that tone presentation alone induces a mild increase in prefrontal gamma $(30-55 \mathrm{~Hz})$ oscillations in saline $(\boldsymbol{A})$, but not MAM $(\boldsymbol{B})$, rats. Control rats that had received a mild footshock paired with a tone display a transient but significant increase in high-frequency activity evoked by the conditioned tone $(\boldsymbol{C})$, that is attenuated in rats that had been exposed to the tone before the conditioning period (E). In contrast, MAM-treated rats did not display a robust response to the conditioned tone either in rats with $(\boldsymbol{F})$ or without $(\boldsymbol{D})$ previous tone exposure. The horizontal line depicts the $2 \mathrm{~s}$ tone presentation whereas the dashed line represents the control response (i.e., no tone presentation); $n=3-5$ rats/group.

after ketamine administration (Cunningham et al., 2006). In addition, a disruption in the recruitment of PV-positive neurons has been shown to alter pyramidal cell activity and oscillatory network function in a conditional GluR-A ${ }^{\text {PVCre-1- }}$ knock-out mouse (Fuchs et al., 2007). These alterations in network activity were reportedly associated with impairments in spatial working memory and exploratory behavior, suggesting that PV interneuron recruitment is required for the expression of key aspects of animal behavior (Fuchs et al., 2007). Indeed, the failure of a conditioned stimulus to evoke gamma activity in these regions in MAM-treated rats suggests that the ability to associate a stimulus with an event, either neutral or noxious, is impaired.

Functional imaging studies show that schizophrenia patients consistently fail to activate the mPFC and vHipp during cognitive tasks (Heckers et al., 1998; Meyer-Lindenberg et al., 2001; Perlstein et al., 2001; Weiss and Heckers, 2001; Barch et al., 2003; Preston et al., 2005), and abnormalities have been reported in induced theta- and gamma-band activity in frontal regions during a number of different paradigms, including cognitive processes such as working memory (Haig et al., 2000; Ford et al., 2002; Gonzalez-Hernandez et al., 2003; Spencer et al., 2003;
Schmiedt et al., 2005; Cho et al., 2006; Basar-Eroglu et al., 2007). Moreover, these deficits in synchronous activity are significantly correlated with reduced task performance in schizophrenia patients, further demonstrating the role for coordinated network activity in the expression of cognitive behaviors. Consistent with this premise, we now report that deficits in mPFC and vHipp oscillatory activity are associated with an impaired behavioral expression of latent inhibition in MAM-treated rats. Thus, control rats that had been exposed to the tone before the conditioning period demonstrated a significantly attenuated prefrontal oscillatory response to the tone in both the theta and gamma frequencies that was correlated with a robust latent inhibition, i.e., an attenuated conditioned response to the tone. In contrast, MAM-treated rats displayed a deficit in the behavioral expression of latent inhibition, with previous exposure to the tone having no significant effect on the locomotor response to the conditioned stimulus (Flagstad et al., 2005). We found that this was correlated with an inability to activate cortical assemblies in response to the conditioned tone in MAM-treated rats.

Together, the data presented here are consistent with evidence for a reduction in prefrontal and hippocampal activation during task performance in schizophrenia and animal models of psychosis. Of course, these studies focus primarily on changes in neuronal activity during task performance that does not necessarily reflect the baseline activity of the structure. Indeed, there is significant and increasing evidence for enhanced activity within the hippocampus of schizophrenia patients at rest (Heckers et al., 1998; Malaspina et al., 1999, 2008; Medoff et al., 2001; Lewandowski et al., 2005; Tamminga et al., 2008).

Evidence for baseline hippocampal hyperactivity was first reported in the medial temporal lobe in human schizophrenia patients using single photon emission computed tomography (SPECT) imaging (Malaspina et al., 1999). More recently, techniques with higher spatial resolution have provided evidence for increased regional cerebral blood flow $\left(\mathrm{H}_{2} \mathrm{O}^{15}\right.$-PET) (Tamminga et al., 2008) and increased regional cerebral blood volume (dynamic susceptibility contrast MRI) (Malaspina et al., 2008) at rest in schizophrenia patients. Furthermore, the hyperactivity within these hippocampal subfields is highly correlated with clinical measures of psychosis and delusions (Malaspina et al., 2008; Tamminga et al., 2008) suggesting that aberrant hippocampal activity may be a key anatomical substrate involved in the positive symptoms of schizophrenia.

We reported previously that a pathologically enhanced activity within the vHipp actually drives the dopamine dysfunction in MAM-treated rats (Lodge and Grace, 2007). Thus, MAM-treated rats demonstrate increased DA neuron population activity (i.e., the number of neurons firing spontaneously). This is attributed 
to hyperactivity within the ventral hippocampus since TTX inactivation of the vHipp normalized both the augmented DA neuron activity and behavioral hyperresponsivity to amphetamine administration (Lodge and Grace, 2007). Given the pathological decrease in PV neuron density within the vSub reported in the current study, we propose that a decrease in intrinsic GABAergic function may be the origin of the hippocampal hyperactivity purported to underlie the dopamine dysfunction in psychosis (Lodge and Grace, 2007; Lisman et al., 2008).

The data presented here are consistent with evidence for a reduction in PVcontaining interneurons that is correlated with altered prefrontal and hippocampal activation during task performance in schizophrenia and animal modes of psychosis. Furthermore, the inability to activate cortical assemblies is consistent with the impairments in cognitive function that are a hallmark of the disease. As we have emphasized previously (Lodge and Grace, 2007), we are aware that gestational MAM administration is not necessarily an accurate recapitulation of the etiology of schizophrenia in humans. Nonetheless, we posit that at the core of this disorder is a disruption of systems interactions that can be modeled in animals, but when placed in the context of complex human brain and behavioral patterns, yields the complex pattern of psychopathology recognized as schizophrenia. Such an understanding of the functional interactions among these systems and how disruption within these circuits affects information processing is central to gaining a better understanding of disease. Moreover, these results suggest that a more effective pharmacotherapeutic strategy may lie in the restoration of interneuron regulation of patterned activity within ventral hippocampal circuits.

\section{References}

Abdul-Monim Z, Neill JC, Reynolds GP (2007) Subchronic psychotomimetic phencyclidine induces deficits in reversal learning and alterations in parvalbumin-immunoreactive expression in the rat. J Psychopharmacol 21:198-205.

Abercrombie M (1946) Estimation of nuclear population from microtome sections. Anat Rec 94:239-247.

Akbarian S, Kim JJ, Potkin SG, Hagman JO, Tafazzoli A, Bunney WE Jr, Jones EG (1995) Gene expression for glutamic acid decarboxylase is reduced without loss of neurons in prefrontal cortex of schizophrenics. Arch Gen Psychiatry 52:258-266.

Baeg EH, Kim YB, Jang J, Kim HT, Mook-Jung I, Jung MW (2001) Fast spiking and regular spiking neural correlates of fear conditioning in the medial prefrontal cortex of the rat. Cereb Cortex 11:441-451.

Barch DM, Sheline YI, Csernansky JG, Snyder AZ (2003) Working memory and prefrontal cortex dysfunction: Specificity to schizophrenia compared with major depression. Biological Psychiatry 53:376-384.

Bartos M, Vida I, Frotscher M, Meyer A, Monyer H, Geiger JR, Jonas P (2002) Fast synaptic inhibition promotes synchronized gamma oscillations in hippocampal interneuron networks. Proc Natl Acad Sci U S A 99:13222-13227.
Bartos M, Vida I, Jonas P (2007) Synaptic mechanisms of synchronized gamma oscillations in inhibitory interneuron networks. Nat Rev Neurosci 8:45-56.

Basar-Eroglu C, Brand A, Hildebrandt H, Karolina Kedzior K, Mathes B, Schmiedt C (2007) Working memory related gamma oscillations in schizophrenia patients. Int J Psychophysiol 64:39-45.

Behrens MM, Ali SS, Dao DN, Lucero J, Shekhtman G, Quick KL, Dugan LL (2007) Ketamine-induced loss of phenotype of fast-spiking interneurons is mediated by NADPH-oxidase. Science 318:1645-1647.

Benes FM, Berretta S (2001) GABAergic interneurons: Implications for understanding schizophrenia and bipolar disorder. Neuropsychopharmacology 25:1-27.

Benes FM, Lim B, Matzilevich D, Walsh JP, Subburaju S, Minns M (2007) Regulation of the GABA cell phenotype in hippocampus of schizophrenics and bipolars. Proc Natl Acad Sci U S A 104:10164-10169.

Berretta S, Lange N, Bhattacharyya S, Sebro R, Garces J, Benes FM (2004) Long-term effects of amygdala GABA receptor blockade on specific subpopulations of hippocampal interneurons. Hippocampus 14:876-894.

Buzsáki G, Leung LW, Vanderwolf CH (1983) Cellular bases of hippocampal EEG in the behaving rat. Brain Res 287:139-171.

Cho RY, Konecky RO, Carter CS (2006) Impairments in frontal cortical gamma synchrony and cognitive control in schizophrenia. Proc Natl Acad Sci U S A 103:19878-19883.

Cunningham MO, Hunt J, Middleton S, LeBeau FE, Gillies MJ, Gillies MG, Davies CH, Maycox PR, Whittington MA, Racca C (2006) Region- 

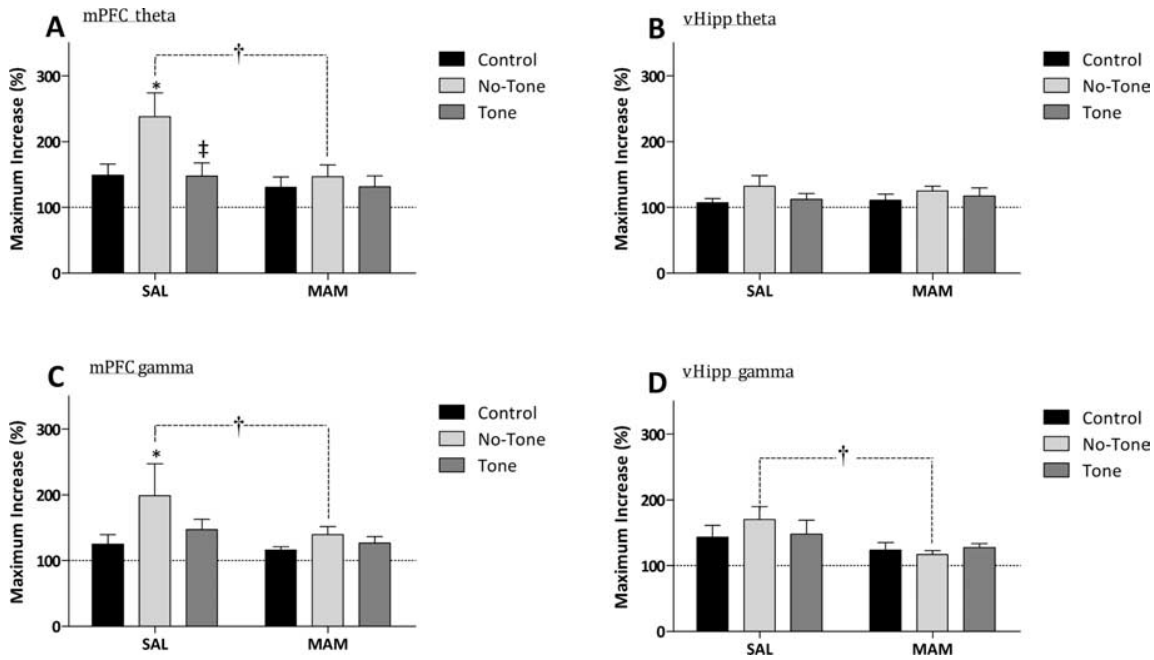

Figure 11. MAM-treated rats display a significantly attenuated oscillatory activity during latent inhibition training and testing. Analysis of the maximum increase in oscillatory activity demonstrates significant deficits in the induction of $\mathrm{mPFC}$ theta $(A)(4-12$ $\mathrm{Hz})$, mPFC gamma (C) $(30-55 \mathrm{~Hz})$, vHipp gamma (D) $(30-55 \mathrm{~Hz})$, but not vHipp theta $(\boldsymbol{B})(4-12 \mathrm{~Hz})$ between saline and MAM-treated rats. * Significant difference compared with no tone trials; ${ }^{*}$ significant difference between tone (dark gray bar) and no tone (light gray bar) pre-exposure; ${ }^{\dagger}$ significant difference between saline and MAM-treated rats ( $p<0.05$, two-way ANOVA with Holm-Sidak post hoc: $n=3-5$ rats/group).

specific reduction in entorhinal gamma oscillations and parvalbuminimmunoreactive neurons in animal models of psychiatric illness. J Neurosci 26:2767-2776.

Flagstad P, Mørk A, Glenthøj BY, van Beek J, Michael-Titus AT, Didriksen M (2004) Disruption of neurogenesis on gestational day 17 in the rat causes behavioral changes relevant to positive and negative schizophrenia symptoms and alters amphetamine-induced dopamine release in nucleus accumbens. Neuropsychopharmacology 29:2052-2064.

Flagstad P, Glenthøj BY, Didriksen M (2005) Cognitive deficits caused by late gestational disruption of neurogenesis in rats: a preclinical model of schizophrenia. Neuropsychopharmacology 30:250-260.

Ford JM, Mathalon DH, Whitfield S, Faustman WO, Roth WT (2002) Reduced communication between frontal and temporal lobes during talking in schizophrenia. Biol Psychiatry 51:485-492.

Freund TF, Katona I (2007) Perisomatic inhibition. Neuron 56:33-42.

Fuchs EC, Zivkovic AR, Cunningham MO, Middleton S, Lebeau FE, Bannerman DM, Rozov A, Whittington MA, Traub RD, Rawlins JN, Monyer H (2007) Recruitment of parvalbumin-positive interneurons determines hippocampal function and associated behavior. Neuron 53:591-604.

González-Hernández JA, Cedeño I, Pita-Alcorta C, Galán L, Aubert E, Figueredo-Rodríguez P (2003) Induced oscillations and the distributed cortical sources during the Wisconsin card sorting test performance in schizophrenic patients: new clues to neural connectivity. Int J Psychophysiol 48:11-24.

Goto Y, Grace AA (2006) Alterations in medial prefrontal cortical activity and plasticity in rats with disruption of cortical development. Biol Psychiatry 60:1259-1267.

Gourevitch R, Rocher C, Le Pen G, Krebs MO, Jay TM (2004) Working memory deficits in adult rats after prenatal disruption of neurogenesis. Behav Pharmacol 15:287-292.

Grace AA, Moore H (1998) Regulation of information flow in the nucleus accumbens: a model for the pathophysiology of schizophrenia. In: Origins and development of schizophrenia: advances in experimental psychopathology (Lenzenweger MF, Dworkin RH, eds), pp 123-157. Washington, DC: American Psychological Association.

Haig AR, Gordon E, De Pascalis V, Meares RA, Bahramali H, Harris A (2000) Gamma activity in schizophrenia: evidence of impaired network binding? Clin Neurophysiol 111:1461-1468.

Harte MK, Powell SB, Swerdlow NR, Geyer MA, Reynolds GP (2007) Deficits in parvalbumin and calbindin immunoreactive cells in the hippocampus of isolation reared rats. J Neural Transm 114:893-898.

Hashimoto T, Volk DW, Eggan SM, Mirnics K, Pierri JN, Sun Z, Sampson AR, Lewis DA (2003) Gene expression deficits in a subclass of GABA neurons in the prefrontal cortex of subjects with schizophrenia. J Neurosci 23:6315-6326.

Heckers S, Rauch SL, Goff D, Savage CR, Schacter DL, Fischman AJ, Alpert NM (1998) Impaired recruitment of the hippocampus during conscious recollection in schizophrenia. Nat Neurosci 1:318-323.

Heckers S, Stone D, Walsh J, Shick J, Koul P, Benes FM (2002) Differential hippocampal expression of glutamic acid decarboxylase 65 and 67 messenger RNA in bipolar disorder and schizophrenia. Arch Gen Psychiatry 59:521-529.

Lavin A, Moore HM, Grace AA, Lavin A, Moore HM, Grace AA (2005) Prenatal disruption of neocortical development alters prefrontal cortical neuron responses to dopamine in adult rats. Neuropsychopharmacology 30:1426-1435.

Le Pen G, Gourevitch R, Hazane F, Hoareau C, Jay TM, Krebs MO (2006) Peri-pubertal maturation after developmental disturbance: A model for psychosis onset in the rat. Neuroscience 143:395-405.

Lewandowski NM, Schobel SS, Wu WE, Corcoran C, Malaspina D, Small SA (2005) Isolating hippocampal subregions most vulnerable to schizophrenia. Soc Neurosci Abstr 31:443.1.

Lewis DA, Lund JS (1990) Heterogeneity of chandelier neurons in monkey neocortex: corticotropin-releasing factor- and parvalbumin-immunoreactive populations. J Comp Neurol 293:599-615.

Lewis DA, Hashimoto T, Volk DW (2005) Cortical inhibitory neurons and schizophrenia. Nat Rev Neurosci 6:312-324.

Lisman JE, Coyle JT, Green RW, Javitt DC, Benes FM, Heckers S, Grace AA (2008) Circuit-based framework for understanding neurotransmitter and risk gene interactions in schizophrenia. Trends Neurosci 31:234-242.

Lodge DJ, Grace AA (2007) Aberrant hippocampal activity underlies the dopamine dysregulation in an animal model of schizophrenia. J Neurosci 27:11424-11430.

Malaspina D, Storer S, Furman V, Esser P, Printz D, Berman A, Lignelli A, Gorman J, Van Heertum R (1999) SPECT study of visual fixation in schizophrenia and comparison subjects. Biol Psychiatry 46:89-93.

Malaspina D, Schobel SS, Lewandowski LM, Corcoran C, Brown T, Small S (2008) Hippocampal dysfunction in cal is associated with schizophrenia. Schizophr Res 102 [Suppl 2]:19-20.

Medoff DR, Holcomb HH, Lahti AC, Tamminga CA (2001) Probing the human hippocampus using rCBF: contrasts in schizophrenia. Hippocampus 11:543-550.

Meyer-Lindenberg A, Poline JB, Kohn PD, Holt JL, Egan MF, Weinberger DR, Berman KF (2001) Evidence for abnormal cortical functional connectivity during working memory in schizophrenia. Am J Psychiatry 158:1809-1817.

Moore H, Jentsch JD, Ghajarnia M, Geyer MA, Grace AA (2006) A neurobehavioral systems analysis of adult rats exposed to methylazoxymethanol acetate on E17: implications for the neuropathology of schizophrenia. Biol Psychiatry 60:253-264.

Paxinos G, Watson C (1986) The rat brain in stereotaxic coordinates. Sydney: Academic.

Penschuck S, Flagstad P, Didriksen M, Leist M, Michael-Titus AT (2006) Decrease in parvalbumin-expressing neurons in the hippocampus and increased phencyclidine-induced locomotor activity in the rat methylazoxymethanol (MAM) model of schizophrenia. Eur J Neurosci 23:279-284.

Perlstein WM, Carter CS, Noll DC, Cohen JD (2001) Relation of prefrontal cortex dysfunction to working memory and symptoms in schizophrenia. Am J Psychiatry 158:1105-1113.

Perry TL, Kish SJ, Buchanan J, Hansen S (1979) Gamma-aminobutyric-acid deficiency in brain of schizophrenic patients. Lancet 1:237-239.

Preston AR, Shohamy D, Tamminga CA, Wagner AD (2005) Hippocampal function, declarative memory, and schizophrenia: anatomic and functional neuroimaging considerations. Curr Neurol Neurosci Rep 5:249-256. 
Reynolds GP, Beasley CL, Zhang ZJ (2002) Understanding the neurotransmitter pathology of schizophrenia: selective deficits of subtypes of cortical GABAergic neurons. J Neural Transm 109:881-889.

Schmiedt C, Brand A, Hildebrandt H, Basar-Eroglu C (2005) Event-related theta oscillations during working memory tasks in patients with schizophrenia and healthy controls. Brain Res Cogn Brain Res 25:936-947.

Spencer KM, Nestor PG, Niznikiewicz MA, Salisbury DF, Shenton ME, McCarley RW (2003) Abnormal neural synchrony in schizophrenia. J Neurosci 23:7407-7411.

Talamini LM, Ellenbroek B, Koch T, Korf J (2000) Impaired sensory gating and attention in rats with developmental abnormalities of the mesocortex. Implications for schizophrenia. Ann N Y Acad Sci 911:486-494.

Tamás G, Buhl EH, Lörincz A, Somogyi P (2000) Proximally targeted GABAergic synapses and gap junctions synchronize cortical interneurons. Nat Neurosci 3:366-371.

Tamminga CA, Ghose S, Thomas B, Mihalakos P, Lu H (2008) Medial temporal cortex pathophysiology in schizophrenia: actions of antipsychotic drugs. Schizophr Res 102 [Suppl 2]:19.

Uhlhaas PJ, Linden DE, Singer W, Haenschel C, Lindner M, Maurer K, Rodriguez E (2006) Dysfunctional long-range coordination of neural ac- tivity during Gestalt perception in schizophrenia. J Neurosci 26:8168-8175.

Uhlhaas PJ, Haenschel C, Nikoliæ D, Singer W (2008) The role of oscillations and synchrony in cortical networks and their putative relevance for the pathophysiology of schizophrenia. Schizophr Bull 34:927-943.

Volk DW, Austin MC, Pierri JN, Sampson AR, Lewis DA (2000) Decreased glutamic acid decarboxylase67 messenger RNA expression in a subset of prefrontal cortical $\gamma$-aminobutyric acid neurons in subjects with schizophrenia. Arch Gen Psychiatry 57:237-245.

Weiss AP, Heckers S (2001) Neuroimaging of declarative memory in schizophrenia. Scand J Psychol 42:239-250.

Whittington MA, Traub RD, Jefferys JG (1995) Synchronized oscillations in interneuron networks driven by metabotropic glutamate receptor activation. Nature 373:612-615.

Woo TU, Whitehead RE, Melchitzky DS, Lewis DA (1998) A subclass of prefrontal gamma-aminobutyric acid axon terminals are selectively altered in schizophrenia. Proc Natl Acad Sci U S A 95:5341-5346.

Zhang ZJ, Reynolds GP (2002) A selective decrease in the relative density of parvalbumin-immunoreactive neurons in the hippocampus in schizophrenia. Schizophr Res 55:1-10. 